Nonlin. Processes Geophys., 20, 85-96, 2013

www.nonlin-processes-geophys.net/20/85/2013/

doi:10.5194/npg-20-85-2013

(C) Author(s) 2013. CC Attribution 3.0 License.

\title{
A Lagrangian approach to the Loop Current eddy separation
}

\author{
F. Andrade-Canto ${ }^{1}$, J. Sheinbaum ${ }^{1}$, and L. Zavala Sansón ${ }^{1}$ \\ ${ }^{1}$ Departmento de Oceanografía Física, CICESE, Carretera Ensenada-Tijuana 3918, 22860 Ensenada, Baja California, México \\ Correspondence to: F. Andrade (fandrade@ cicese.edu.mx)
}

Received: 12 April 2012 - Revised: 27 October 2012 - Accepted: 14 December 2012 - Published: 23 January 2013

\begin{abstract}
Determining when and how a Loop Current eddy (LCE) in the Gulf of Mexico will finally separate is a difficult task, since several detachment re-attachment processes can occur during one of these events. Separation is usually defined based on snapshots of Eulerian fields such as sea surface height ( $\mathrm{SSH}$ ) but here we suggest that a Lagrangian view of the LCE separation process is more appropriate and objective. The basic idea is very simple: separation should be defined whenever water particles from the cyclonic side of the Loop Current move swiftly from the Yucatan Peninsula to the Florida Straits instead of penetrating into the NE Gulf of Mexico. The properties of backward-time finite time Lyapunov exponents (FTLE) computed from a numerical model of the Gulf of Mexico and Caribbean Sea are used to estimate the "skeleton" of flow and the structures involved in LCE detachment events. An Eulerian metric is defined, based on the slope of the strain direction of the instantaneous hyperbolic point of the Loop Current anticyclone that provides useful information to forecast final LCE detachments. We highlight cases in which an LCE separation metric based on SSH contours (Leben, 2005) suggests there is a separated LCE that later reattaches, whereas the slope method and FTLE structure indicate the eddy remains dynamically connected to the Loop Current during the process.
\end{abstract}

\section{Introduction}

One of the most interesting features of the circulation in the Gulf of Mexico (GoM) is the Loop Current (LC). The LC can either extend deep into the northeast GoM $\left(24-28^{\circ} \mathrm{N}\right)$ and turn clockwise back to Cuba and the Florida Straits, or go directly from the Yucatan Channel to the Florida Straits (Candela et al., 2002; Leben, 2005). The LC is well known for shedding large anticyclones (Loop Current Eddies, LCEs) at irregular intervals between 0.5-18.5 months (Sturges and
Leben, 2000), which travel westward across the GoM. These eddies decay in the central Gulf from interaction with other eddies (Lipphardt et al., 2008), or reach the western slope of the GoM and decay by interaction with topography generating coastal currents and eddies (Sturges, 1994).

Although the mechanisms and frequency of LCE shedding have been studied by many authors, critical aspects of the process remain uncertain (Alvera-Azcarate et al., 2009; Maul and Vukovich, 1993; Sturges, 1994; Vukovich, 1995). Some authors have discussed several events where small cyclonic eddies on the periphery of the Loop Current influence the shedding of the LC rings (Fratantoni et al., 1998; Zavala-Hidalgo et al., 2003; Chérubin et al., 2006; Schmitz, 2005). Using $3 \mathrm{yr}$ of direct mooring observations together with altimetry data Athié et al. (2011) find that some shedding events are associated with cyclonic anomalies coming from the Caribbean producing an eastward shift of the LC core. Downstream from the LC, Sturges et al. (2010) finds perturbations in the transport measurements from the cable between Miami and the Bahamas that precede several LCE detachments. All these findings suggest the detachment process is quite complicated, more so, since eddies may frequently detach and reattach from the LC during intrusion into the GoM.

A fundamental tool to study and characterize the detachment process is satellite altimetry. Leben (2005) proposed an LC tracking technique, in which the eddy separation is determined by the breaking of the $17 \mathrm{~cm} \mathrm{SSH}$ contour (17-SSH) considered to mark the LC high velocity core. One problem with this method is that once a separation occurs, it can not determine if the eddy will reattach to the LC later on. Another problem is that when images of sea surface temperature (SST) are also available, SSH and SST fields may provide contradicting information. For instance, in Fig. 1 the SSH image (panel a) indicates an LCE already separated, whereas the SST image (panel b) 

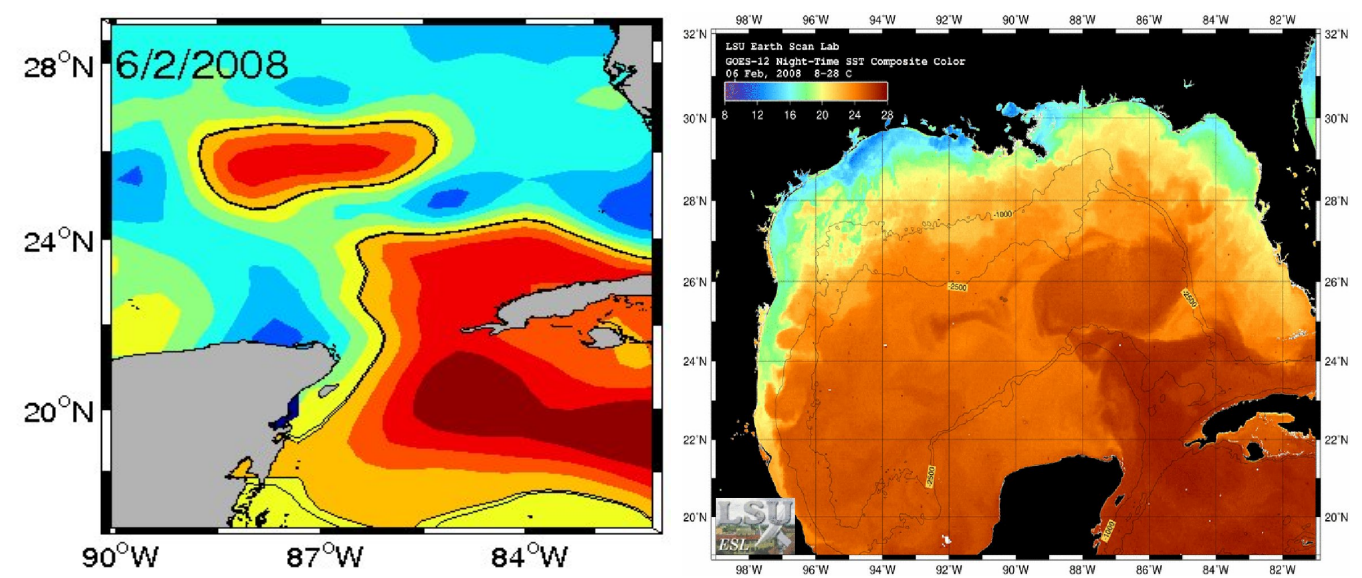

Fig. 1. Weekly snapshot of SSH from AVISO (Archiving, validation and interpretation of satellite oceanographic data) (a), and SST from GOES (Geostationary Operational Environmental Satellite) SST composite produced by Louisiana State University (b), for 6 February 2008. Notice the 17-SSH contour (black line) suggests the Loop Current eddy (LCE) has already detached from the LC whereas the SST composite indicates the LCE is still attached.

suggests the LCE is still attached. Similarly, Fig. 2 shows snapshots of a shedding event highlighting the 17-SSH contour (data computed from AVISO satellite altimetry data, http://www.aviso.oceanobs.com), showing the extension of the LC into the GoM (left panel). A detached LCE is depicted in the middle panel, but, as already mentioned, there is no indication in this SSH field that the eddy would reattach later on, as it happened in this case (right-hand panel). Visual analysis of figures like these might indicate the moment when the shedding begins, but the method does not capture the details of how the process takes place and makes it difficult to determine the final moment of detachment. One reason for this is related to the fact that SSH maps provide an Eulerian view of what is basically a Lagrangian process (Haller, 2005). Besides, since the flow is time-dependent, the SSH field, which plays the role of stream-function for surface geostrophic velocities (in the quasi-gesotrophic framework), does not indicate particle paths in general. Thus, it makes sense to analyze the separation process from a Lagrangian point of view.

Kuznetsov et al. (2002) used near-surface currents from a numerical model and computed material lines to elucidate the interaction between the LC and adjacent eddies. They showed that Lagrangian analysis provides more information about the separation process than the Eulerian point of view, since the Lagrangian approach allows to determine barriers of transport between the rings and the surrounding fluid associated with hyperbolic (saddle) regions in the flow. The evolution and geometry of these manifolds or distinguished material lines provide more information about the separation than the tracking of closed streamlines or vorticity contours in the Eulerian approach. Kuznetsov et al. (2002) suggest that a necessary condition for an LCE separation is the presence of a hyperbolic saddle point below the Loop Current bulge (LCB) and other flow structures. Here we refer to the LCB as the structure that will become the LCE after separation but remains attached or is part of the Loop Current before separation occurs. Lagrangian analysis has also been used to explore vortex pinch-off in laboratory experiments (O'Farrell and Dabiri, 2010) by computing finite time Lyapunov exponents (FTLEs) and identifying Lagrangian coherent structures (LCS). They show that the emergence of new and disconnected LCS from the original or initial LCS that defines the vortex marks the start of the pinch-off process.

In a more recent study, (Branicki and Kirwan, 2010) performed a very detailed 3-D Lagrangian analysis of an LCE (eddy Juggernaut) using FTLEs and defining the eddy boundaries as the intersection of the stable and unstable manifolds of two distinct distinguished hyperbolic trajectories (as defined in Mancho et al., 2006). Lobe dynamics and the socalled turnstile mechanism is used to determine the exchange of fluid between the eddy and its surroundings.

These ideas lead us to propose an alternative method to study the LC shedding process based on particle trajectories whose behaviour can be understood using structures identified in FTLE diagnostics that participate in the LCE separation. In contrast to the detailed analysis of Branicki and Kirwan (2010) and Kuznetsov et al. (2002), our interest is perhaps less ambitious, and is simply to define a Lagrangian LCE separation index that will indicate when fluid particles on the cyclonic side of the LC move directly to the east (Florida Straits) instead of around the LCB inside the GoM. This criterion is objective (i.e., frame independent), and in fact, does not require a precise definition of the LCE boundary, an issue thoroughly analyzed in Branicki and Kirwan (2010). Our hope is that by developing an index that captures part of the Lagrangian aspects of the LCE separation, we will obtain more reliable information about the process than the 

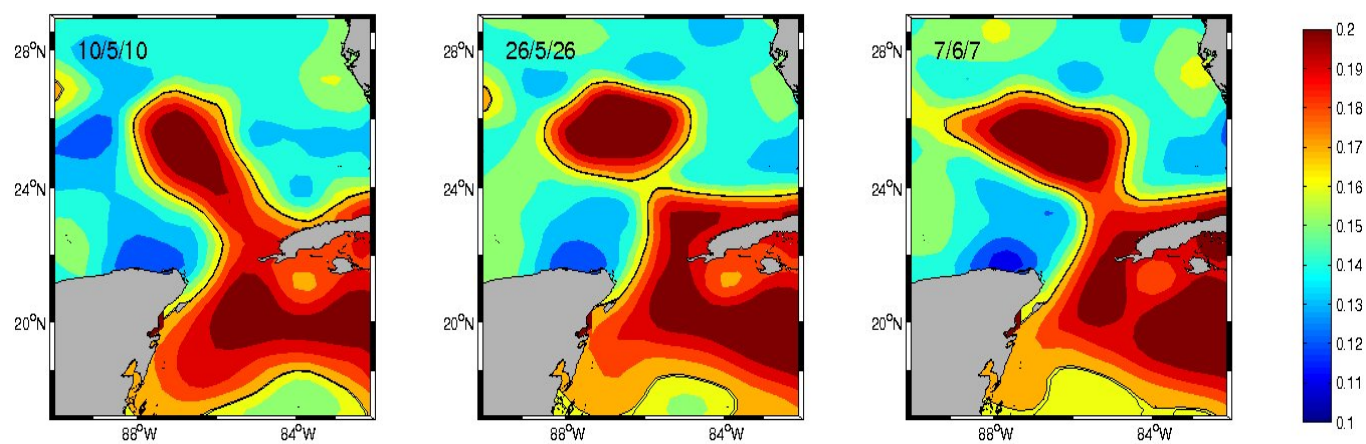

Fig. 2. Weekly snapshots of the SSH field in m for an LCE detachment-reattachment event that occurred in May-June 2008 obtained from AVISO data. Once a separation occurs, the 17-SSH contour does not provide information on whether or not there will be a later reattachment, as it happened in this event.

one provided by snapshots of Eulerian fields such as those based on SSH (Leben, 2005).

To test these ideas we use numerical model output, so the FTLE fields are computed using velocity data from a numerical simulation of the circulation in the Caribbean Sea and GoM based on the NEMO (Nucleus for European Modelling of the Ocean) model (Jouanno et al., 2009). Eight LCE separation cases were analyzed and compared using both the FTLE technique and Leben's SSH contour method (Leben, 2005). The FTLE field was computed for each day, using velocities at a depth of $68 \mathrm{~m}$. Analysis of the FTLE highlights some geometric structures in the flow that actively participate in the detachment process. Although a strictly Lagrangian separation index is not defined, we found that LCB instantaneous hyperbolic saddle points and their strain direction (an Eulerian calculation) provide information consistent with FTLE features such as their local orientation near the stagnation point. An index is defined based on these observations and indicates the orientation of such instantaneous strain directions. It appears to capture the change in particle paths before separation and provides a useful criterion to identify - even predict - that final LCE detachments are about to occur. The LCE separation cases discussed here have particular characteristics that represent several other separation events. By contrasting the FTLE method and Leben's SSH method, we aim to clarify their benefits and limitations.

The paper is organized as follows: Sect. 2 briefly describes the concept of Lagrangian coherent structures, the FTLE method used to obtain them and the data employed to compute the FTLE fields. Section 3 describes the analysis of the FTLE field and develops the new Eulerian metric for LCE separation status based on the local strain orientation; this criterion is compared to the 17-SSH altimetry method. It is shown that the new method is better at determining when the final LCE separation will occur. Section 4 is the summary and conclusions. Appendix A provides mathematical details of some of the calculations.

\section{FTLE}

We use the developed concept of LCS (Haller, 2001a), for example, as regions (or structures of dimension $n-1$, where $n$ is the spatial dimension of the flow field) in unsteady flows based on their stability properties in finite time, which can be roughly detected by looking at the maxima of the finite time Lyapunov exponent diagnostic (see below). The LCS can be considered a generalization of the stable and unstable manifolds using their physical property of being respectively the most repelling and attracting structures for particles located normal to them. Although, in general, they are not strictly material lines (Haller, 2001b and Branicki and Wiggins, 2009), in many instances they provide good approximations to actual material structures (as in the LCE separation problem, see Branicki and Kirwan, 2010). LCS define the boundary between fluid domains of different dynamical characteristics, but in contrast to steady flows, attracting and repelling LCS can intersect each other many times forming lobes, which allow fluid exchange between eddies and the exterior. The study of LCS allows identification of transport barriers, transport mechanisms, and regions of rapid dispersion (Beron-Vera et al., 2008; Shadden et al., 2005, 2006; Mathur et al., 2007; Mancho et al., 2006)

The FTLE method is a useful technique for estimating LCS (Haller, 2001a, 2002; Shadden et al., 2005, 2006; Olascoaga et al., 2006; Lekien and Coulliette, 2007; Lekien et al., 2005, 2007; Mathur et al., 2007) since it provides a measure of the maximum separation rate of initially nearby fluid particles in a finite time. It can be employed in any number of dimensions (Lekien and Coulliette, 2007) but here we focus on the 2-D case. If $\boldsymbol{x}_{0}=\left(x_{0}, y_{0}\right)$ denotes the initial position on a 2-D space of a fluid particle at time $t_{0}$, its position at any time $t$, denoted $\boldsymbol{x}\left(t ; x_{0}, y_{0}\right)$, follows by integrating the trajectory equation:

$\dot{\boldsymbol{x}}=\boldsymbol{u}(x, t)$,

where the overdot stands for time differentiation, and $\boldsymbol{u}=$ $(u, v)$ is the 2-D velocity vector. The FTLE can be defined as 
$\sigma_{t_{0}}^{T}=\frac{1}{2|T|} \ln \lambda_{\max }(\Delta)$

where $\lambda_{\max }$ denotes the maximum eigenvalue of $\Delta$, the finite time Cauchy-Green deformation tensor:

$\Delta\left(t ; x_{0}, t_{0}\right):=\partial_{x_{0}} x\left(t ; x_{0}, t_{0}\right)^{*} \partial_{x_{0}} x\left(t ; x_{0}, t_{0}\right)$,

where $*$ denotes the transpose.

Maps of FTLE are computed by calculating Eqs. (2) and (3) upon estimation of $\partial_{x_{0}} x\left(t_{0}+T ; x_{0}, t_{0}\right)$ by direct finite differentiation of fluid particle trajectories with initial positions distributed on a regular grid.The calculations are performed by using the MANGEN (Manifold Generator) software, a dynamical systems tool-kit designed by F. Lekien and C. Coulliette. Regions of maximum separation rates produce ridges in the FTLE field that approximate attracting (repelling) LCS when integrating particle trajectories backward (forward) in time. Repelling and attracting LCS delineate the boundary between fluid regions with distinct flow characteristics.

The velocity field used to compute the FTLEs is daily model velocity output from a numerical simulation based on Jouanno et al. (2009), in which the NEMO-AGRIF (adaptive mesh refinement software) model was implemented for the Caribbean Sea and GoM $\left(98^{\circ} \mathrm{W}-57^{\circ} \mathrm{W}, 6^{\circ} \mathrm{N}-31^{\circ} \mathrm{N}\right)$. This configuration is a nested grid model with a horizontal resolution of $1 / 15$ degree (approximately $7 \mathrm{~km}$ ) embedded in a coarser grid eddy-permitting ( $1 / 3$ degree, approximately $35 \mathrm{~km}$ resolution) North Atlantic configuration $\left(50^{\circ} \mathrm{N}-20^{\circ} \mathrm{S}\right)$. Both, the coarse and fine resolution grids have 46 vertical levels whose spacing increases from $12 \mathrm{~m}$ at the surface to $250 \mathrm{~m}$ below $1500 \mathrm{~m}$. The two grids interact in both ways using the AGRIF methodology (Debreu, 2000), i.e., the fine grid solution also impacts the solution in the coarse grid. By contrast with the simulation reported in Jouanno et al. (2009), which uses climatological surface forcing, the simulation used here is a $40 \mathrm{yr}$ integration (19582006, $9 \mathrm{yr}$ spin-up) carried out with interannual forcing from the ERA40 reanalysis corrected by Brodeau et al. (2010). As discussed in Jouanno et al. (2009), the model reproduces the mean circulation and eddy characteristics in the region. Although Jouanno et al. (2009) do not discuss the circulation in the GoM, preliminary results of the inter-annual run (19582006) by Sheinbaum et al. (2010) indicate the simulation of LC eddy shedding events required for the present study are consistent with observations.

The daily output of velocity and SSH fields corresponds to years 1997 to 2005 in which we identified eight shedding events. The basic difference among them is whether or not one or several reattachments occur once an LCE separates. The following discussion focuses particularly on two of the eight events analyzed in which this difference is more evident. The initial separation time in all events is first identified from the model SSH field.
The velocity used to advect the particles corresponds to the sixth vertical level of the model at $68 \mathrm{~m}$ depth. This depth is chosen close to the surface but at the same time below the mean mixed layer depth so that some ageostrophic components are partially filtered out to facilitate future comparison of the results with those obtained from surface geostrophic velocities from altimetry data. Results, however, are similar if a shallower level is chosen (not shown). The vertical velocity at this depth is not exactly zero, but sufficiently small that horizontal velocities can be approximated as non-divergent. Several studies (Olascoaga et al., 2006; Kuznetsov et al., 2002) suggest that this is a reasonable approximation which properly describes transport processes for the space and time scales relevant to the LCE separation. According to Branicki and Kirwan (2010) this assumption is valid if the product of $H \epsilon$ is much smaller than unity, where $\epsilon$ is the average vertical shear in a fluid layer of thickness $H$ divided by the average horizontal velocity. As in Branicki and Kirwan (2010), we find the criterion is satisfied in a layer about $150-200 \mathrm{~m}$ wide (from the surface) since $H \epsilon \ll 1$, which supports the original assumption.

Particle trajectories are determined using a time-stepadapting fourth/fifth-order Runge-Kutta method scheme with a fixed $1 \mathrm{~h}$ time step and a tricubic method for the required spatio-temporal interpolation of the numerically generated velocity field to the particle positions. The initial particle "grid" is four times finer than the velocity grid and is composed of $1000 \times 1000$ particles. To evaluate Eq. (2), MANGEN uses central differences and standard eigenvalue solvers, see Shadden et al. (2005); Lekien and Coulliette (2007) for details. Daily FTLE maps are computed for a period of 30 days, advecting particles backward in time 35 days, i.e., integrating Eq. (1) from $t(i)$ to $t(i-35)$, where $i=1,2, \ldots 30$ indicates starting day of integration. This period captures the shedding events including detachments and reattachments if they occur, and also keeps a large number of the initial particles in the region of interest. The attractingrepelling properties of the LCSs change with time and are only valid for the period they are calculated from. This means that results could change if a different integration period is employed (Branicki and Kirwan, 2010), and in fact, some structures may even change their properties within the time interval used in their computation. Recent work defines LCS cores (Olascoaga and Haller, 2012) as structures that preserve their attracting-repelling character during the whole interval. Our FTLE analysis is qualitative and such detailed calculations are not needed. In that sense, we find that results are not sensitive to slight modifications of the integration period (even if it is extended to 50 days, similar features in the FTLE diagnostics, their character and evolution are identified, see below). 

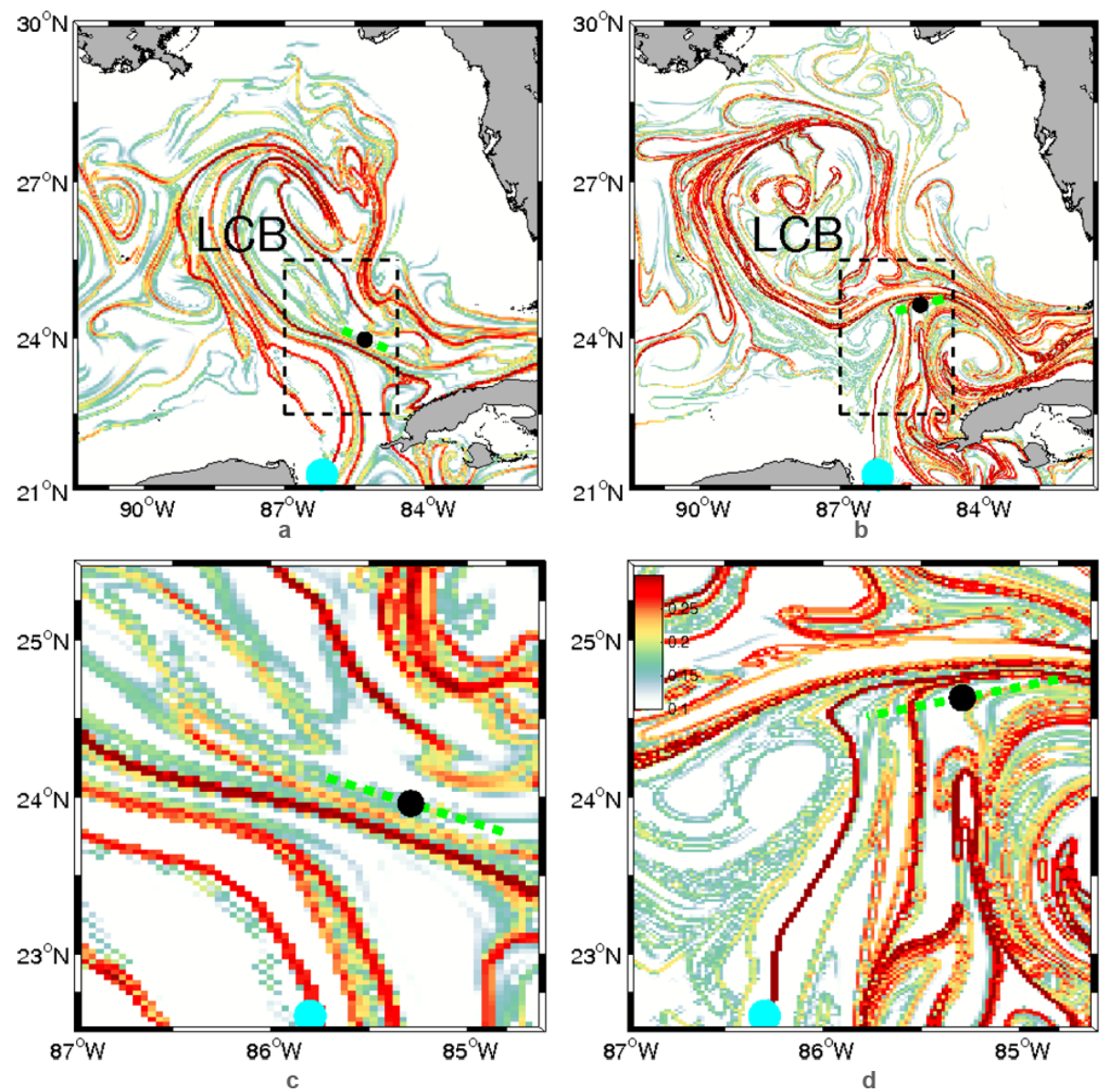

Fig. 3. Snapshots of FTLE field in $\mathrm{d}^{-1}$ computed backward in time for 35 days, for 1 June (a) and 30 June (b) of model simulation year 2000. Regions of red tones indicate the possible attracting LCSs (ridges). These images show the Loop Current Bulge (LCB) in conditions where the LCB is attached (a) and separating (b). Both panels show the instantaneous hyperbolic point (black dot) computed from the "frozen" velocity field of each day together with its strain direction (dashed green segment), named LCBsd in the text. The cyan dot marks the origin of a ridge on the western side of the Yucatan Ridge (YR), which plays an important role in the separation process. To better appreciate the ridges involved, (c) and (d) zoom into the region indicated by rectangles shown in (a) and (b) (see text for details).

\section{Results}

Figure 3 shows the FTLE fields computed backward in time for 35 days for 1 June (Fig. 3a) and 30 June 2000 (Fig. 3b). The regions with intense red tones indicate maximum values of the FTLEs and identify possible attracting LCS, so we refer to them simply as ridges. This is a rough estimate but sufficient for our calculations, since properties of the identified FTLE ridge structures are corroborated a posteriori by looking at the behaviour of particles (see below). Notice that ridges in the FTLE field not necessarily represent hyperbolic LCSs, as they may correspond to regions of high shear (Haller, 2011).

The two top panels depict a full view of the FTLE field. Several highly complex and entangled structures are observed, but some particular coherent features relevant to the LCE separation can be identified. These are discussed below using Fig. $3 \mathrm{c}$ and d, zooming into the saddle region.
In Fig. 3a and b the LCB is located close to the center of the map and can be identified by low FTLE values which are surrounded by high FTLE values. Below the LCB, a black dot marks its associated instantaneous hyperbolic point (LCBhp) and the dashed green line indicates its corresponding strain direction (LCBsd). The circulation in the region is such that several instantaneous stagnation points can be found at any given time, though it is not always possible to relate one of them to the LCB. The method used to locate the LCBhp is explained in the Appendix A and uses Eulerian information from SSH, and Okubo-Weiss fields. It is clear in these figures that the LCBhp is in a region surrounded by FTLE ridges and that the LCBsd (green segment) has roughly the same orientation as the FTLE ridges near the saddle point.

A cyan circle at the lower margin in Fig. 3 highlights the origin of a ridge associated with the western Yucatan Current. The intense FTLE values of this ridge can be associated to 

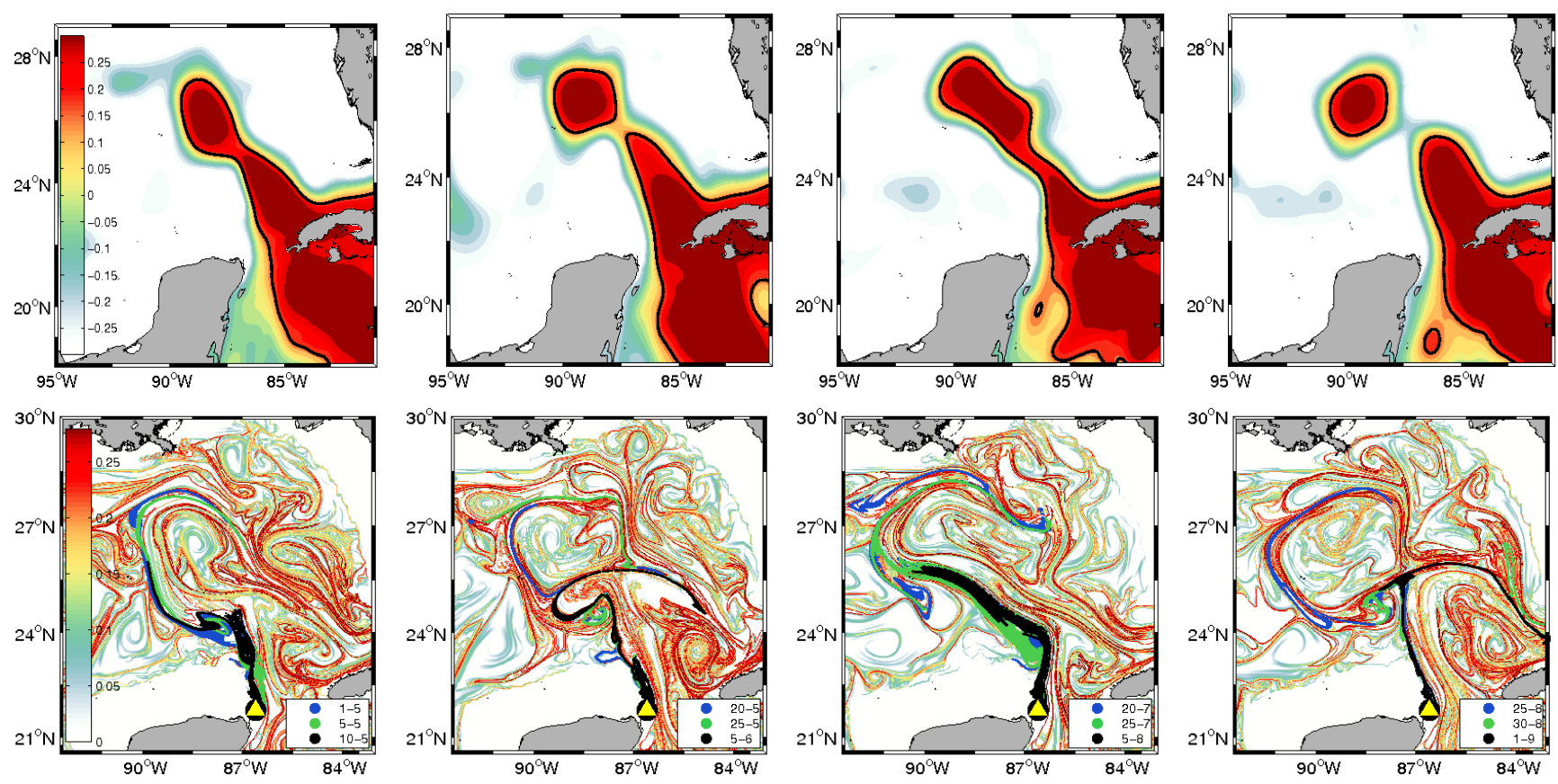

Fig. 4. Snapshots of SSH fields in $\mathrm{m}$ (top panel) and FTLE fields in $\mathrm{d}^{-1}$ (lower panel) for the days indicated in Fig. $6 a$ : 19 May, 9 June, 9 August and 15 September. The SSH field in the second column shows a detached LCE while the FTLE is highly structured and the YR still wraps around the LCB. Most of the particles (blue to black dots, the color indicates the day in which they are settled; marked in the bottom of each snapshot) seeded on the left corner of the Yucatan Peninsula go north following the YR, (yellow triangle) indicating the eddy is still attached. Finally, in the last panel, a substantial number of particles travel directly to the east following the YR, signalling that the LCE is totally separated. (see text).

the LC. We name it the Yucatan ridge (YR) from its origin, but one can see the YR marks the external rim of the LC. It extends northward surrounding the LCB and then joins the Florida Current in Fig. 3a. By contrast, in Fig. 3b the YR turns eastward and no longer wraps around the LCB which is now nearly separated from the Loop Current forming the LCE.

Differences between these two conditions of the LCE separation process can be better appreciated in Fig. 3c and d, which zoom into the saddle point region marked by the square in Fig. 3a and b. In Fig. 3c, high FTLE values are visible between the ridges parallel to the LCBsd and the YR indicating the presence of other ridges, whereas in Fig. 3d, low FTLE values are found between these two ridges. These low FTLE values are related to particles which remain together (separate less rapidly) approaching the ridges, i.e., particles which have their origin on the western (cyclonic) side of the Yucatan and Loop Currents are now moving northeastward attracted by the YR (actually nearby particles on both sides of the YR move towards it). Notice also the different orientations of the strain direction in the figures. The LCBsd is oriented in the northwest-southeast direction in Fig. 3a, whereas in Fig. 3b, its orientation is southwest-northeast. This change in strain direction when the LCE is about to separate was identified in the eight shedding events studied in this work and will be used later on to define a separation index.

In Fig. 3, we find that changes in the structure of YR, as time evolves, indicate when fluid particles on the cyclonic side of the Yucatan Current will start to move eastward instead of going to the northwest around the LCB. This is a more reliable indicator of LCE separation than tracking a particular contour of the sea surface height, simply because in a time dependent flow, particle paths are not the same as stream lines. The SSH field (streamlines for the surface flow in the quasi-geostrophic limit) may indicate that an LCE has detached, but particles may still move deep into the Gulf around the LCB, as will be shown below. Monitoring the YR and using it to define separation is not that simple, due to its Lagrangian character.

We can, however, formulate a relatively simple LCE Eulerian separation index based on the observed changes in the slope of the instantaneous strain direction at the LCB hyperbolic point, which appears to capture qualitative properties of the Lagrangian behaviour discussed above.

The lower panels in Figs. 4 and 5 show seeded particles (green, black and blue colors) on top of FTLE diagnostics, and were obtained from snapshots of movies of FTLEs and particle trajectories in two particular events during 1997 and 2002. These animations suggest quite clearly that particles are indeed attracted and follow some of the previously 

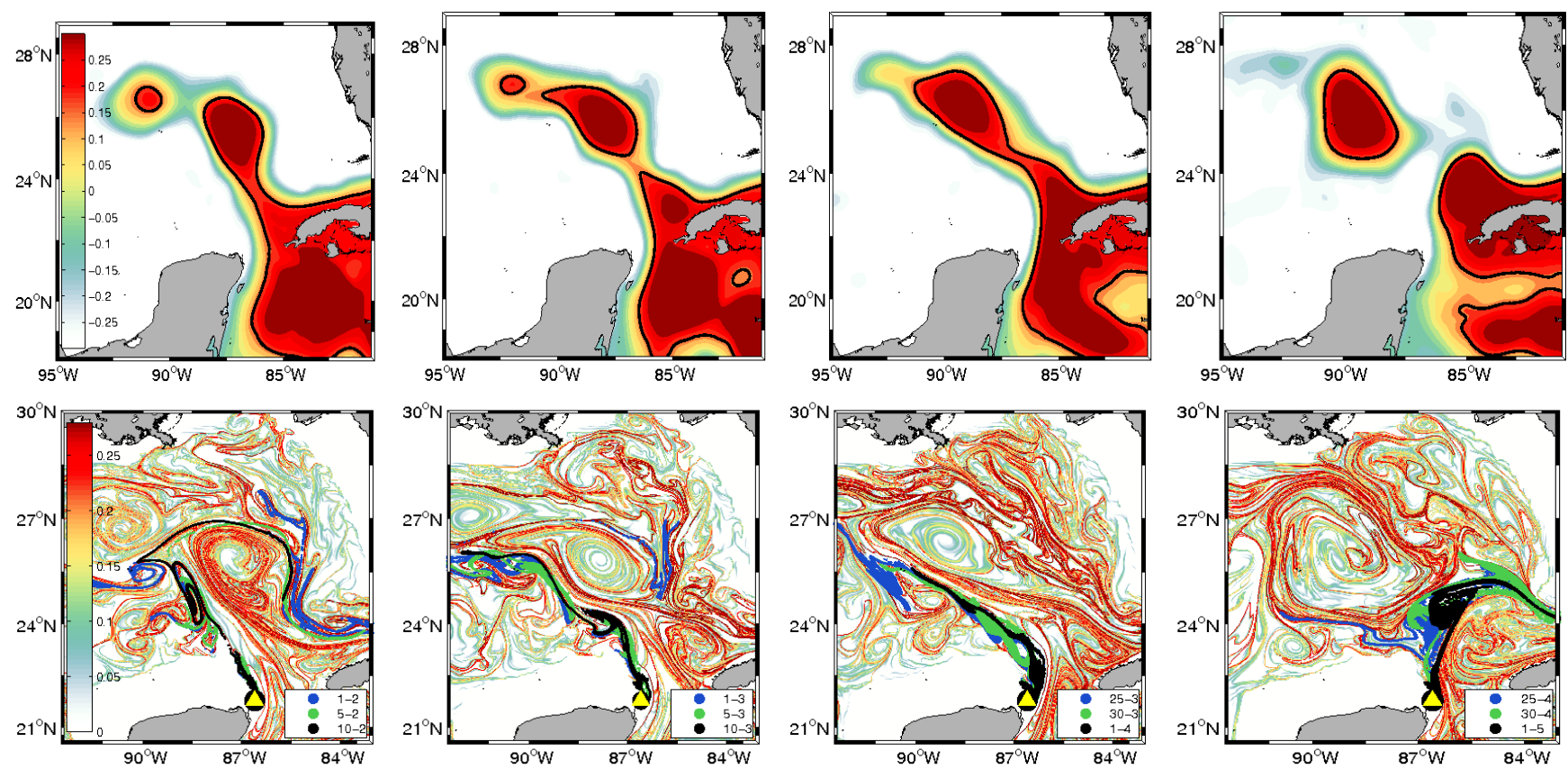

Fig. 5. Snapshots of SSH fields in $m$ (top panel) and FTLE fields in $d^{-1}$ (lower panel) for the days indicated in Fig. 6c: 21 February, 19 March, 17 April and 12 May. SSH maps for March and April suggest the LCE separated while the FTLE maps and the particle trajectories clearly indicate there is no direct eastward intrusion of particles from the western side of the Yucatan Current until the final snapshot, where the LCE is totally separated (see text).

identified ridges. Particularly interesting is the YR, whose evolution does seem to control how particles on the cyclonic side of the YC-LC behave. What we found, is that when the YR wraps around the LCB, marking also the movement of the seeded particles, the LCBsd has a distinctive (negative) northwest-southeast orientation. A soon as the YR intrudes to the east, this orientation begins to change until it becomes positive and strongly southwest-northeast before an LCE separation. As shown below (Fig. 6), the index's change of sign from negative to positive does indicate that a final LCE separation will soon occur, but no particular range of values can be used to determine whether or not the eddy has separated. The fact that the LCBsd is, in general, parallel to an FTLE ridge and that one can relate it to particle movement, makes it a quasi-Lagrangian separation index.

The change in LCBsd orientation is calculated by locating the instantaneous hyperbolic stagnation point and the eigenvalues and eigenvectors of the gradient velocity tensor for each day at the point. The slope of the LCBsd is given by the orientation of eigenvector $\mathbf{V}^{+}$corresponding to the largest eigenvalue $\lambda^{+}$of $\nabla \mathbf{U}$. Negative values indicate that the LCBsd is oriented in the northwest-southeast direction, which means the LCB is attached, whereas positive values indicate southwest-northeast orientation and the LCE is close to separation. Several stagnation points appear in the flow at any given time, so in order to identify the actual hyperbolic point related to the LCB, a method was developed to reduce the uncertainty generated by the presence of other hyperbolic points (for details of all these calculations see Appendix A). It should be mentioned that finding the LCBhp is not always possible, though this only happens on few occasions.

A question also arises as to whether the time scales involved in these calculations and those related to the LCE separation are consistent, so as to guarantee that the fields evolve sufficiently slowly. Such a condition allows one to relate the trajectory of the instantaneous hyperbolic stagnation point and its velocity Jacobian eigenvalue-eigenvector pairs to the presence of a nearby Lagrangian hyperbolic trajectory. Although we are not interested in computing the hyperbolic trajectory, the conditions established (Haller et al., 1997, 1998; Velasco Fuentes, 2001) that permit this connection are satisfied in all separation cases (see Appendix A).

In order to quantify the merits of the new LCE separation metric, we compare it to the SSH contour-length method for the same shedding periods in the model simulation. We identify eight different separation events, which are: May-September of 1997, July-August 1998, MayJuly 2000, August-November 2001, February-May 2002, July-September 2003, February-June 2004, and AprilJune 2005. The events of 1998, 2000, 2001, and 2005 are quite similar and both metrics yield similar results. Figure 6 shows the evolution of the LCBsd slope (continuous line), and that of the contour length (17-SSH) metric (dashed line) for the events in years 1997, 1998, 2002, 2003 and 2004. The 


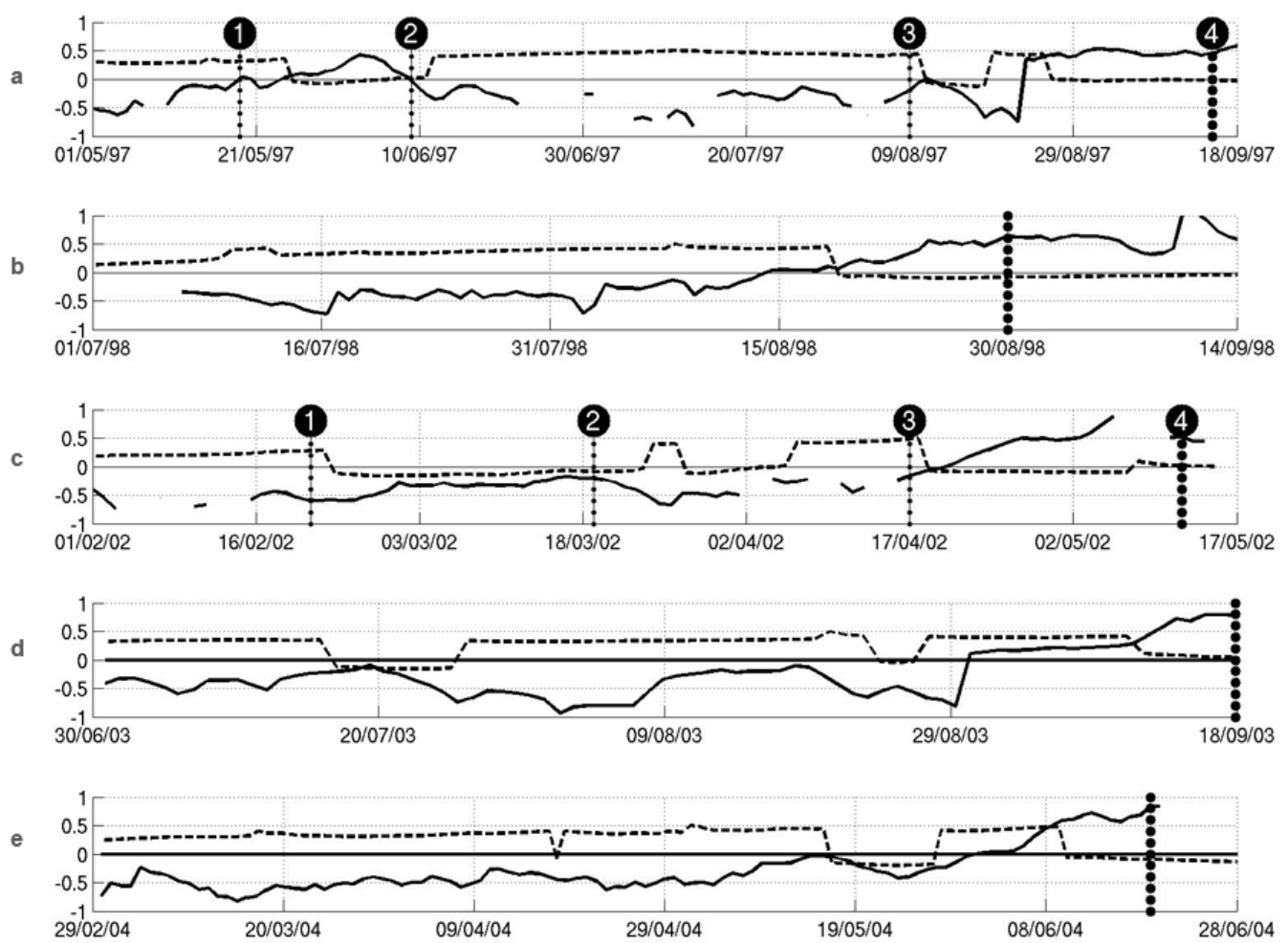

Fig. 6. Time series of two indices used to determine the LC state and LCE separation. The dashed line is the length of the 17-SSH contour whereas the continuous line depicts the LCBsd slope index. Each panel represents conditions during 5 different shedding periods of the numerical simulation corresponding to May-August 1997, June-August 1998, February-April 2002, July-September 2003, and MarchJune 2004, respectively. The vertical dashed line indicates the final separation date determined from analysis of the FTLE field (see text). Numbers and vertical dotted lines in (a) and (c) are explained in Figs. 4 and 5. Drops in the SSH index suggest the LCE is separated and remains in that stage as long as the value of this index remains small. The slope index indicates there will be an LCE detachment once its sign changes from negative to positive. Note that on several occasions the SSH index suggests the eddy is separated whilst the slope index indicates the eddy is still connected (see text).

vertical dotted lines indicate the time of final separation determined from visual inspection of the FTLE fields, and is chosen as the time when a lobe bounded by the YR (and a substantial number of seeded particles) has intruded directly east and as far as $84^{\circ} \mathrm{W}$. Numbered lines in Fig. 6a and c indicate the times of SSH and FTLE snapshots discussed in Figs. 4 and 5. The 17-SSH contour was chosen by Leben (Leben, 2005) to represent the outer boundary of the LC, so that high or increasing values of its length indicate the $\mathrm{LC}$ is growing, and sudden drops in its length suggest an LCE has separated.

Figure 6a, c, d, and e show various detachmentreattachment events according to this index (dashed line) and its sudden drops. By contrast, the slope index has negative values throughout several of these "detachments", indicating there has been no separation in the Lagrangian sense. Particularly interesting is the 2002 event (Fig. 6c), where the SSH index suggests the LCE separated on the third week of February and remained so for about two months, whereas the slope index shows negative values throughout this period.
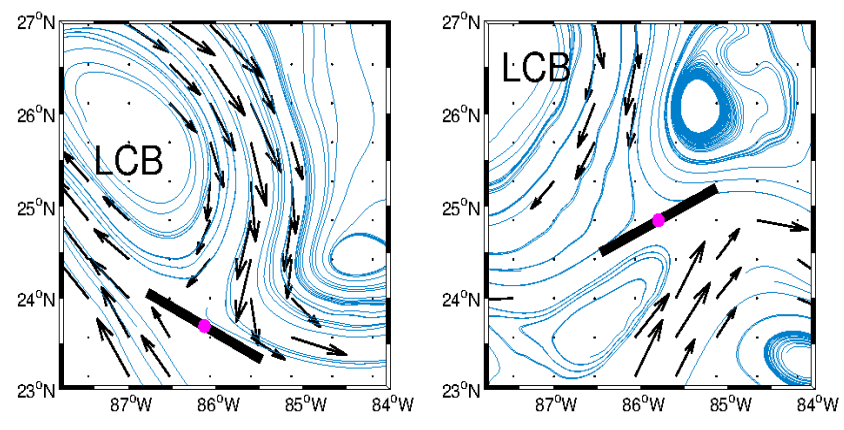

Fig. 7. Summary of the two different stages of the Loop Current eddy (LCE) separation process: (a) before separation; (b) after separation. The blue lines are the streamlines, the black arrows are the velocity vectors, the black line is the LCBsd associated with the LCBhp (dot in magenta). The orientation of the strain directions is determined by the relative positions of the LCB and the LC (see text). 


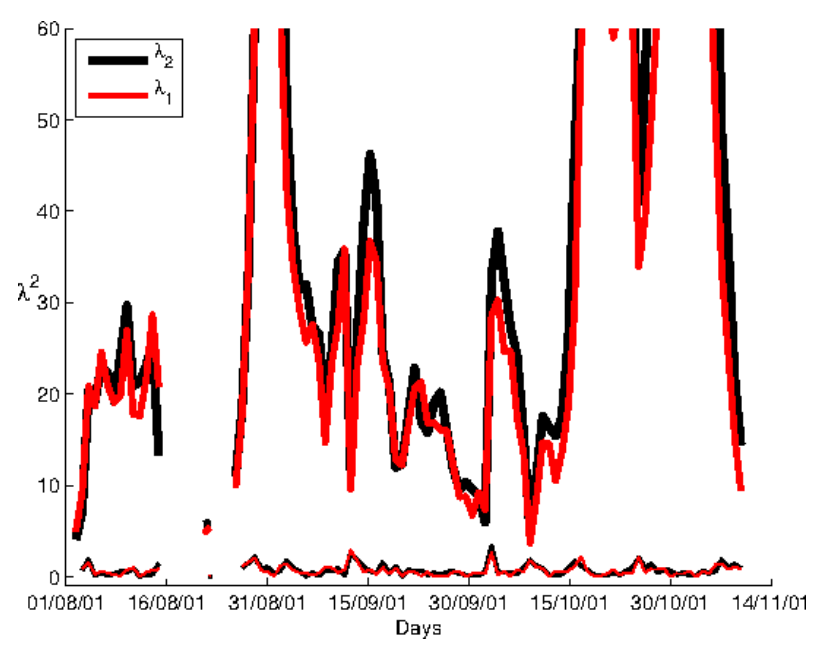

Fig. 8. Time evolution of the strength of the hyperbolicity $\lambda_{2,1}^{2}$ (thick lines), and its rate of change $\lambda_{1.2}^{\prime}$ (thin lines). The computations were made for the evolution of the LCBph 2001 event.

To demonstrate that the slope index provides a closer indication of Lagrangian LCE separation, we analyze in more detail the 1997 and 2002 separation events by looking at different snapshots of the SSH, and FTLEs in Figs. 4 and 5, for the days marked in Fig. 6a and c. Besides this, passive particles were seeded on the west side of the Loop Current and advected by the flow. Their position is plotted together with the FTLEs to corroborate the interpretation of the computed ridges, as structures that attract nearby particles and form the "skeleton" of the flow.

In Fig. 4, the second column of panels corresponding to snapshot 2 (9 June 1997), shows that while the SSH field depicts a separated eddy (top panel), the FTLE field (lower panel) is highly structured and the YR marked by the yellow triangle, though quite folded, ceases to wrap the LCB. Notice that some particles do cross to the east directly from Yucatan. This may be the reason why the slope index (continuous line in Fig. 6a) has a positive value and yet the LCB is almost separated. Nonetheless, a movie of the process (not shown) indicates the Cuban anticyclone (the structure below the LCB) begins to decrease and this allows reattachment of the LCB. Looking now at the fourth column of panels, with conditions representing what we identify with true final LCE separation time, we see that a substantial number of particles travel directly east without going around the LCB. The key feature here, is that the YR is the boundary of a thin lobe that separates the LC and LCE structures, an intrusion clearly marked by the particles reaching $84^{\circ} \mathrm{W}$ and which prompts our definition of LCB separation. A lobe that intrudes less to the east may still retreat, and the LCE separation will not continue.

The separation event of simulation year 2002, shown in Fig. $6 c$, is perhaps more striking in terms of differences between separation criteria, since the drop in the SSH index indicates an LCE separation that starts 20 February and lasts for about two months (dashed line), whilst the slope index remains negative indicating no detachment at all (continuous line). In Fig. 5, the SSH maps (top panel) suggest a separated eddy from snapshot 2 onwards, whereas the FTLE maps and particle trajectories clearly indicate there is no eastward intrusion of particles directly from the western side of the Yucatan and Loop Currents until the final snapshot. Observe that in this case, the lobe formed by the YR is quite wide on the western side, wraps around the LC and extends to $83.5^{\circ} \mathrm{W}$.

Relative vorticity maps (not shown), indicate that some ridges coincide with the rims of eddies and main currents where vorticity changes sign, though the FTLEs are much more complex. The FTLE maps are calculated backward in time and represent a kind of Lagrangian evolution history, so instantaneous vorticity and FTLE ridges do not need to agree, though there is clearly a connection between them that needs to be explored further.

\section{Summary and conclusions}

Fig. 6a and c indicate that the SSH index can be somewhat misleading if we define LCE separation in a Lagrangian framework. Nevertheless, it is an index easy to calculate from data that provides very useful information. Our purpose here has been to clarify its content and complement it with indices that provide some sort of Lagrangian information. FTLE maps are quite complex, but clearly indicate the structures involved in the separation process, particularly the role played by what we call the Yucatan Ridge, YR, which marks the "road" followed by particles from the western or cyclonic side of the Yucatan and Loop Currents. When these particles move swiftly to the east joining the Florida Current, we say the LCE has separated.

Recent work (Olascoaga and Haller, 2012; Haller-BeronVera, 2012; Madrid et al., 2009) improves on criteria and computational methods to actually determine the location and evolution of LCS cores, a useful extension of saddle points to finite-time flow data. These methods could clearly be applied to better understand the Lagrangian character of the LCE separation problem. Meanwhile, we devised an Eulerian (quasi-Lagrangian) index based on calculating the instantaneous hyperbolic point associated to the LCLCE and its strain direction. Negative slopes (northwestsoutheast) indicate the LCE is attached, whereas positive slopes (southwest-northeast) generally mean the LCE is in the process of separation. Even though, a case was found (1997 separation event, Fig. 4) in which positive slopes did not indicate near-future LCE separation. However, the SSH index failed many more times than the slope index by wrongly indicating LCE separations when in Lagrangian terms the LCEs were still attached. 
The slope index is associated with the LCE separation because its sign indicates the relative positions of the eddy and the LC before and after the separation process takes place. These positions determine the strain directions that eventually lead to the vortex separation. This is shown in Fig. 7, which shows snapshots of the conditions involved before and at LCE separation in two typical simulations. Before separation, the influence of the LC at the western side of the hyperbolic point is directed to the northwest, while the flow on the eastern side points to the southeast. As a result, the slope of the strain direction is negative. In contrast, when the eddy is separated the LC is directed to the northeast, while the LCB is moving to the southwest. Consequently, the slope of the strain direction becomes positive.

The hyperbolic point determines the advection properties in the saddle region: particles which are initially located a short distance from the stagnation point will approach it and move away from it along the strain direction. Olascoaga and Haller (2012) show that they provide predictive information on flow instabilities that change the behaviour of tracer patches before they are fully developed. In our case, computing the instantaneous hyperbolic point and its slope orientation allowed us to predict if passive particles closest to the hyperbolic point will move north, surrounding the LC anticyclonic center (therefore LCE attachment) or if they will move east directly to the Florida Straits (LCE separation).

Although the method has only been applied to eight numerically simulated shedding periods, the main results indicate that the slope index is a good indicator of the Loop Current status that should also work with other models and geostrophic velocities derived from altimetry data. It is clear, therefore, that FTLE or similar Lagrangian methods seem more adequate and reliable to analyze, describe and determine the LCE separation.

\section{Appendix A}

Due the presence of several eddies in the region, a method must be used to determine the instantaneous hyperbolic point related to the Loop Current Bulge (main anticyclonic center) that we called the LCBhp in the text. To do this, first we have to identify the hyperbolic region in the frozen time velocity field associated to the LCB, that is characterized by the contraction and stretching of the flow. This is done by using the following steps:

- The gross characteristics of the region, where the hyperbolic point appears during the shedding process, are initially determined looking at any of the dynamical fields involved (including $\mathrm{SSH}$ ) and selecting the area where separation occurs, which is between $89-83^{\circ} \mathrm{W}$ and $23-$ $28^{\circ} \mathrm{N}$ in our experiments.
- Velocity data are filtered in both directions to eliminate small scale features since we are searching for the hyperbolic point related to a large scale structure (the LCB). The zero of the SSH surrounds the anticyclonic structures implicated in the shedding process. Thus, all velocities outside this contour are set to zero for the calculation described next.

- Hyperbolic regions are related to intense strain and/or deformation. Therefore, the Okubo-Weiss parameter, which identifies regions where vorticity or strain are dominant (Okubo, 1970; Weiss, 1991), is calculated within the separation region to help define where the LCBhp may be located. The Okubo-Weiss parameter $W$ is defined as

$W=s_{\mathrm{n}}^{2}+s_{\mathrm{s}}^{2}-\zeta^{2}$,

where $s_{\mathrm{n}}, s_{\mathrm{s}}$, and $\zeta$ are the normal and shear components of strain, and the relative vorticity of the flow, defined by

$s_{\mathrm{n}}=\frac{\partial u}{\partial x}-\frac{\partial v}{\partial y}, \quad s_{\mathrm{s}}=\frac{\partial v}{\partial x}+\frac{\partial u}{\partial y}, \quad \zeta=\frac{\partial v}{\partial x}-\frac{\partial u}{\partial y}$,

where $x$ and $y$ are standard cartesian coordinates, $u$ and $v$ are their velocity components and we assume the 2-D velocity vector is non-divergent.

The parameter $W$ separates a non-divergent twodimensional flow in two different regions: a vorticitydominated region $(W<0)$, and a strain-dominated region $(W>0)$. Maximum values of the Okubo-Weiss parameter indicate regions where the strain is high and we relate this to the hyperbolic region of the LCB for the frozen time velocity field.

- Once the hyperbolic region is identified, the instantaneous hyperbolic point is found using the methodology described in Velasco Fuentes and Marinone (1999) to determine the stagnation points for each time slice (the velocity field for each day). The method computes those cells where zeros of the velocity field are likely to occur, which are cells where neither $u_{i j}$ nor $v_{i j}$ have the same sign at the four corners $\left(u_{i j}\right.$ and $v_{i j}$ being zonal and meridional velocities on the grid respectively).

Properties of the stagnation points are given by the eigenvalues and eigenvectors of $\nabla \mathbf{U}$ at that point:

$\nabla \mathbf{U}=\left[\begin{array}{ll}\frac{\partial U}{\partial x} & \frac{\partial U}{\partial y} \\ \frac{\partial V}{\partial x} & \frac{\partial V}{\partial y}\end{array}\right]$

which is evaluated using centered finite-differences. If the two eigenvalues of this matrix are real and of opposite sign, the stagnation point is then a saddle or hyperbolic point. The positive (negative) eigenvalueeigenvector pair give the strain (contraction) direction properties. 
The method described above is Eulerian by nature, and Mancho et al. (2006) show that one must be careful not to derive or interpret the results from these calculations in general Lagrangian terms. Haller et al. (1998) define criteria that guarantees one can relate properties of the instantaneous strain direction with the presence of a hyperbolic trajectory. Figure 8 shows a plot of the time series of the square of the velocity Jacobian eigenvalues and their time-derivative for the separation event of 2001. For slowly evolving velocity fields, the latter should be smaller than the eigenvalues squared and this is satisfied in all our experiments. Other conditions involving the eigenvector matrix are also satisfied. In our case, it turns out that the LCE instantaneous strain direction appears to be parallel to the ridges of the FTLE. The LCE separation involves well defined mesoscale structures, and their saddle regions/points of interest are relatively slowly evolving and spatially confined. This may explain why the slope index works.

Acknowledgements. We would like to thank an anonymous reviewer for the insightful comments that led to substantial improvements to the manuscript. Fruitful discussions and advice from Javier Berón-Vera, Josefina Olascoaga, and Oscar Velasco are deeply appreciated. Thanks to Bob Leben for providing us with his Loop Current metrics toolbox and F. Lekien for making freely available his MANGEN code. Altimetry data were produced by Salto/Duacs and distributed by Aviso (http://www.jason.oceanobs.com), with support from CNES. The model configuration was set up between CICESE and the Drakkar Project (www.ifremer.fr/lpo/drakkar) This work was supported by CICESE's core funding.

Edited by: A. M. Mancho

Reviewed by: two anonymous referees

\section{References}

Alvera-Azcarate, A., Barth, A., and Weisberg, R.: The surface circulation of the Caribbean Sea and the Gulf of Mexico as inferred from satellite altimetry, J. Phys. Oceanogr., 39, 640-657, 2009.

Athié, G., Candela, J., Sheinbaum, J., and Ochoa, J.: Preconditioning of the Loop Current behaviour by the Mexican Caribbean Circulation, J. Geophys. Res., 117, C03018, doi:10.1029/2011JC007090, 2011.

Beron-Vera, F., Olascoaga, M., and Goni, G.: Oceanic mesoscale eddies as revealed by Lagrangian coherent structures, Geophys. Res. Lett., 35, L12603, doi:10.1029/2008GL033957, 2008.

Branicki, M. and Kirwan, D.: Stirring: The Eckard paradigm revisited, Int. J. Eng. Sci., 458, 1027-1042, 2010.

Branicki, M. and Wiggins, S.: Finite-time Lagrangian transport analysis: stable and unstable manifolds of hyperbolic trajectories and finite-time Lyapunov exponents, Nonlin. Processes Geophys., 17, 1-36, doi:10.5194/npg-17-1-2010, 2010.

Brodeau, L., Barnier, B., Treguier, A., Penduff, T., and Gulev, S.: An ERA40-based atmospheric forcing for global ocean circulation models, Ocean Model., 31, 88-104, 2010.
Candela, J., Sheinbaum, J., Ochoa, J., Badan, A., and Leben, R.: The potential vorticity flux through the Yucatan Channel and the Loop Current in the Gulf of Mexico, Geophys. Res. Lett., 29, 16-1-16-4, doi:10.1029/2002GL015587,2002.

Chérubin, L., Morel, Y., and Chassignet, E.: Loop Current ring shedding: the formation of cyclones and the effect of topography, J. Phys. Oceanogr., 36, 569-591, 2006.

Debreu, L.: Raffinement adaptatif de maillage et méthode de zoom, Application aux modeles d'océan, Ph.D. thesis, Université Joseph Fourier, Grenoble, France, 2000.

Fratantoni, P., Lee, T., Podesta, G., and Muller-Karger, F.: The influence of Loop Current perturbations on the formation and evolution of Tortugas eddies in the southern Straits of Florida, J. Geophys. Res-Ocean., 103, 24759, doi:10.1029/98JC02147, 1998.

Haller, G.: Distinguished material surfaces and coherent structures in three-dimensional fluid flows, Physica D, 149, 248-277, 2001a.

Haller, G.: Lagrangian structures and the rate of strain in a partition of two-dimensional turbulence, Phys. Fluids, 13, 3365, doi:10.1063/1.1403336, 2001b.

Haller, G.: Lagrangian coherent structures from approximate velocity data, Phys. Fluids., 14, 1851, doi:1851,10.1063/1.1477449, 2002.

Haller, G.: An objective definition of a vortex, J. Fluid Mech., 525, 1-26, 2005.

Haller, G.: A variational theory of hyperbolic Lagrangian Coherent Structures, Physica D, 240, 574-598, 2011.

Haller, G. and Beron-Vera, F. J.: Geodesic theory of transport barriers in two-dimensional flows, Physica D, 241, 1680-1702, 2012.

Haller, G. and Poje, A. C.: Eddy growth and mixing in mesoscale oceanographic flows, Nonlin. Processes Geophys., 4, 223-235, doi:10.5194/npg-4-223-1997, 1997.

Haller, G. and Poje, A. C.: Finite time transport in aperiodic flows, Physica D, 119, 352-380, 1998.

Jouanno, J., Sheinbaum, J., Barnier, B., and Molines, J.: The mesoscale variability in the Caribbean Sea, Part II: Energy sources, Ocean Model., 26, 226-239, 2009.

Kuznetsov, L., Toner, M., Kirwan, J., Jones, C., Kantha, L., and Choi, J.: The loop current and adjacent rings delineated by Lagrangian analysis of the near-surface flow, J. Mar. Res., 60, 405429, 2002.

Leben, R.: Altimeter-derived loop current metrics, Geophys. Monogr., Amer. Geophys. Union, 161, 181-202, 2005.

Lekien, F. and Coulliette, C.: Chaotic stirring in quasi-turbulent flows, Philos. T. R. Soc. A., 365, 3061-3084, 2007.

Lekien, F., Coulliette, C., Mariano, A., Ryan, E., Shay, L., Haller, G., and Marsden, J.: Pollution release tied to invariant manifolds: A case study for the coast of Florida, Physica D, 210, 1-20, 2005.

Lekien, F., Shadden, S., and Marsden, J.: Lagrangian coherent structures in n-dimensional systems, J. Math. Phys., 48, 065404, doi:10.1063/1.1477449, 2007.

Lipphardt, B., Poje, A., Kirwan, A., Kantha, L., and Zweng, M.: Death of three Loop Current rings, J. Mar. Res., 66, 25-60, 2008.

Madrid, J. A. J. and Mancho, A. M.: Distinguished trajectories in time dependent vector fields, Chaos, 19, 013111, doi:10.1063/1.3056050, 2009.

Mancho, A., Small, D., and Wiggins, S.: A tutorial on dynamical systems concepts applied to Lagrangian transport in oceanic flows defined as finite time data sets: Theoretical and computa- 
tional issues, Physics Reports, 437, 55-124, 2006.

Mathur, M., Haller, G., Peacock, T., Ruppert-Felsot, J., and Swinney, H.: Uncovering the Lagrangian skeleton of turbulence, Phys. Rev. Lett., 98, 144502, doi:10.1103/PhysRevLett.98.144502, 2007.

Maul, G. and Vukovich, F.: The relationship between variations in the Gulf of Mexico Loop Current and Straits of Florida volume transport, Proc. Natl. Aca. Sci. Phys. Oceanogr., 23, 785-796, 1993.

Okubo, A.: Horizontal dispersion of floatable particles in the vicinity of velocity singularities such as convergences, Deep Sea Res. Oceanogr. Abstr., 17, 445-454, 1970.

Olascoaga, M. and Haller, G.: Forecasting sudden changes in environmental pollution patterns, Proc. Natl. Acad. Sci., 109, 47384743, 2012.

Olascoaga, M., Rypina, I., Brown, M., Beron-Vera, F., Kocak, H., Brand, L., Halliwell, G., and Shay, L.: Persistent transport barrier on the West Florida Shelf, Geophys. Res. Lett., 33, L22603, doi:10.1029/2006GL027800, 2006.

O'Farrell, C. and Dabiri, J.: A Lagrangian approach to identifying vortex pinch-off, Chaos, 20, 017513-017513, 2010.

Schmitz, W.: Cyclones and westward propagation in the shedding of anticyclonic rings from the Loop Current, Geophys. Monogr. Ser., 161, 241-261, doi:10.1029/161GM18, 2005.

Shadden, S., Lekien, F., and Marsden, J.: Definition and properties of Lagrangian coherent structures from finite-time Lyapunov exponents in two-dimensional aperiodic flows, Physica D, 212, 271-304, 2005.
Shadden, S., Dabiri, J., and Marsden, J.: Lagrangian analysis of fluid transport in empirical vortex ring flows, Phys. Fluids., 18, 047105, doi:10.1063/1.2189885, 2006.

Sheinbaum, J., Perez-Brunius, P., Lopez, J., Barnier, B., and Molines, J.: Circulation in the Gulf of Mexico from a 40 year run of a two-way nested NEMO-AGRIF numerical model, Ocean Sciences Meeting, 2010.

Sturges, W.: The frequency of ring separations from the Loop Current, J. Phys. Oceanogr., 24, 1647-1651, 1994.

Sturges, W. and Leben, R.: Frequency of ring separations from the Loop Current in the Gulf of Mexico: A revised estimate, J. Phys. Oceanogr., 30, 1814-1819, 2000.

Sturges, W., Hoffmann, N., and Leben, R.: A Trigger Mechanism for Loop Current Ring Separations, J. Phys. Oceanogr., 40, 900 913, 2010.

Velasco Fuentes, O. U.: Chaotic advection by two interacting finitearea vortices, Phys. Fluids, 13, 901-912, doi:10.1063/1.1352626, 2001.

Velasco Fuentes, O. U. and Marinone, S.: A numerical study of the Lagrangian circulation in the Gulf of California, J. Mar. Syst., 22, 1-12, 1999.

Vukovich, F.: An updated evaluation of the Loop Current's eddyshedding frequency, J. Geophys. Res., 100, 8655-8655, 1995.

Weiss, J.: The dynamics of enstrophy transfer in two-dimensional hydrodynamics, Physica D, 48, 273-294, 1991.

Zavala-Hidalgo, J., Morey, S., and O'Brien, J.: Cyclonic eddies northeast of the Campeche Bank from altimetry data, J. Phys. Oceanogr., 33, 623-629, 2003. 